\title{
diffusion-fundamentals.org
}

The Open-Access Journal for the Basic Principles of Diffusion Theory, Experiment and Application

\section{Gold nanostructure assisted thermophoretic trapping of single nano-objects}

\author{
Marco Braun*, Frank Cichos \\ Molecular Nanophotonics Group, Institute for Experimental Physics I, Universität Leipzig, \\ Leipzig, Germany \\ *marco.braun@uni-leipzig.de
}

The manipulation and trapping of nano-objects that undergo Brownian motion are of great interest in soft-matter sciences. Optical tweezing is the most common technique for the trapping of individual particles in solution and is based on the optical gradient force. Hence, a sufficiently high polarizability of the particle in the solution is required. While it is thus easy to trap single dielectric particles larger than $100 \mathrm{~nm}$, a trapping of smaller objects such as single molecules by means of optical tweezers can hardly be realized. Molecular trapping can be achieved by a technique called Anti-Brownian Electrokinetic trap (ABEL trap) [1], which exploits the feedback-controlled electric field of four electrodes. Hence, the latter technique requires electrical contacts, which introduce difficulties when fabricating multiple traps. Here, we present an all-optical technique which replaces the electric fields by highly localized thermal fields. The so-called thermophoretic trap exploits thermophoretic interactions of a particle and a liquid when applying a temperature gradient, which e.g. locally distorts the screening of the surface charges and by that induce a particle drift sufficient for the manipulation of small nanoparticles or molecules [2].
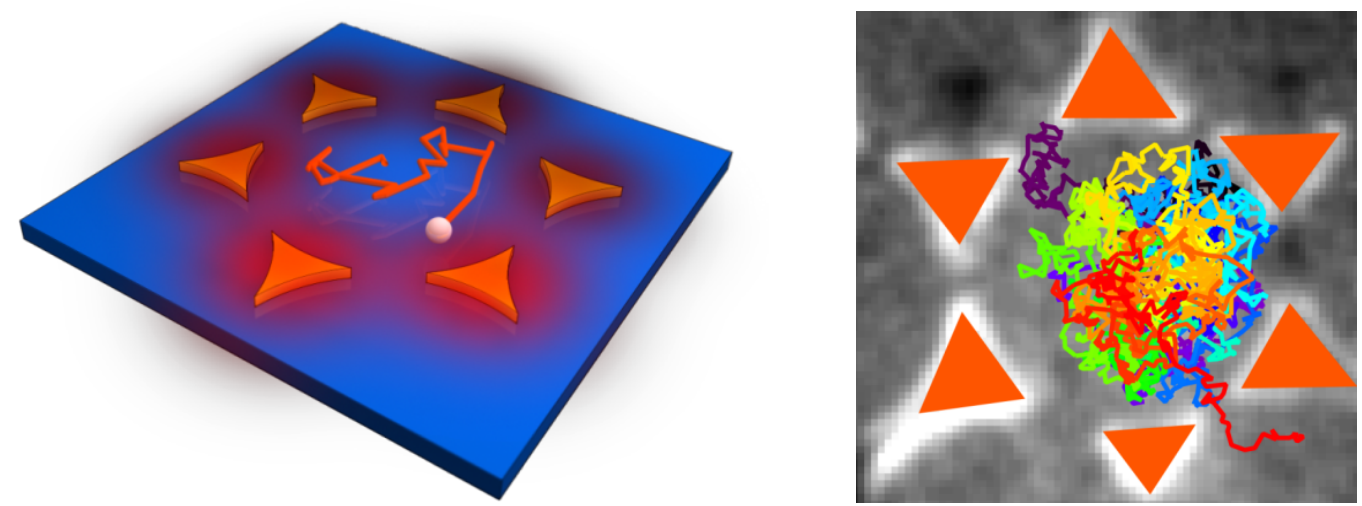

Figure 1: (left) Scheme of the thermophoretic trap consisting out of triangle-shaped Au islands (right) Trajectory of a trapped polystyrene sphere

In our approach the temperature field is generated by optically heated Au nanostructures fabricated by microsphere lithography (Fig. 1) [3]. Due to the small dimensions of the heat sources, even a small temperature increase introduces large temperature gradients causing a strong thermophoretic drift by which the motion of a diffusing particle can be manipulated.

\section{References}

[1] A.E. Cohen, W.E. Moerner: Method for trapping and manipulating nanoscale objects in solution. Appl. Phys. Lett. 86, 093109 (2005)

[2] S. Duhr, D. Braun: Why molecules move along a temperature gradient. PNAS 103, 19678-19682 (2006)

[3] Y. Li, W. Cai, G. Duan: Ordered micro/nanostructured arrays based on the monolayer colloidal crystals. Chem. Mater. 120, 615-624 (2008) 